Systematic Review

\title{
The Effectiveness of Manual Therapy for Relieving Pain, Stiffness, and Dysfunction in Knee Osteoarthritis: A Systematic Review and Meta-Analysis
}

Qinguang $\mathrm{Xu}, \mathrm{MD}^{1,2}$, Bei Chen, MD³,4 Yueyi Wang, MD², Xuezong Wang, MD³ Dapeng Han, MD, Daofang Ding, $\mathrm{PhD}^{1,3}$, Yuxin Zheng, MD ${ }^{1,3}$, Yuelong Cao, MD, PhD ${ }^{1,3}$, Hongsheng Zhan, MD, PhD ${ }^{3}$, and Yao Zhou, MD²

From: ${ }^{1}$ Institute of Arthritis Research, Shanghai Academy of Chinese Medical Sciences, Guanghua Integrative Medicine Hospital/Shanghai University of Traditional Chinese Medicine, China; ${ }^{2}$ Department of Orthopedics and Traumatology, Fenghua Hospital of Traditional

Chinese Medicine, Ningbo, China; ${ }^{3 S h i ' s ~ C e n t e r ~ o f ~}$ Orthopedics and Traumatology, Shuguang Hospital, Shanghai University of Traditional Chinese

Medicine, China; ${ }^{4}$ Department of Chinese Traditional Traumatology, Baoshan Hospital of Integrated Traditional Chinese and Western Medicine, China

Address Correspondence: Yuelong Cao, MD, PhD Institute of Arthritis Research, Shanghai Academy of Chinese Medical Sciences, Guanghua Integrative Medicine Hospital/ Shanghai University of Traditional Chinese Medicine, China

Email: ningtcm@126.com

Disclaimer: This work was supported by the National Natural Science Foundation of People's Republic of China (NO: 81373665). Drs. Xu and Chen contributed equally to this manuscript.

Manuscript received: 05-24-2016 Revised manuscript received: 12-04-2016

Accepted for publication: 12-30-2016

Free full manuscript: www.painphysicianjournal.com
Background: Knee osteoarthritis (KOA) is the most common form of arthritis, leading to pain disability in seniors and increased health care utilization. Manual therapy is one widely used physical treatment for KOA.

Objective: To evaluate the effectiveness and adverse events (AEs) of manual therapy compared to other treatments for relieving pain, stiffness, and physical dysfunction in patients with KOA.

Study Design: A systematic review and meta-analysis of manual therapy for KOA.

Methods: We searched PubMed, EMBASE, the Cochrane Library, and Chinese databases for relevant randomized controlled trials (RCTs) of manual therapy for patients with KOA from the inception to October 2015 without language restrictions. RCTs compared manual therapy to the placebo or other interventional control with an appropriate description of randomization. Two reviewers independently conducted the search results identification, data extraction, and methodological quality assessment. The methodological quality was assessed by PEDro scale. Pooled data was expressed as standard mean difference (SMD), with 95\% confident intervals (Cls) in a random effects model. The meta-analysis of manual therapy for KOA on pain, stiffness, and physical function were conducted.

Results: Fourteen studies involving $841 \mathrm{KOA}$ participants compared to other treatments were included. The methodological quality of most included RCTs was poor. The mean PEDro scale score was 6.6. The meta-analyses results showed that manual therapy had statistically significant effects on relieving pain (standardized mean difference, SMD $=-0.61,95 \% \mathrm{Cl}-0.95$ to $-0.28, P$ $=76 \%)$, stiffness (SMD $=-0.58,95 \% \mathrm{Cl}-0.95$ to $-0.21, P=81 \%)$, improving physical function $(\mathrm{SMD}=-0.49,95 \% \mathrm{Cl}-0.76$ to $-0.22, P=65 \%)$, and total score $(\mathrm{SMD}=-0.56,95 \% \mathrm{Cl}-0.78$ to $-0.35, P=50 \%)$. But in the subgroups, manual therapy did not show significant improvements on stiffness and physical function when treatment duration was less than 4 weeks. And the long-term information for manual therapy was insufficient.

Limitations: The limitations of this systematic review include the paucity of literature and inevitable heterogeneity between included studies.

Conclusion: The preliminary evidence from our study suggests that manual therapy might be effective and safe for improving pain, stiffness, and physical function in KOA patients and could be treated as complementary and alternative options. However, the evidence may be limited by potential bias and poor methodological quality of included studies. High-quality RCTs with longterm follow-up are warranted to confirm our findings.

Key words: Knee osteoarthritis, manual therapy, systematic review

Pain Physician 2017; 20:229-243 
O steoarthritis (OA) is the most common form of arthritis, and the leading cause of disability and pain affecting middle-aged and elderly people worldwide $(1,2)$, caused by structural changes in joints resulting in pain, deterioration of function, and disability $(3,4)$. In Western countries, most people over 65 years of age suffer from this disease (5), and it is particularly common in the knee $(6,7)$. Current conventional treatments include non-pharmacological measures, medication, and surgical procedures. According to recommendations by the American College of Rheumatology (ACR), the pharmacological treatment of knee osteoarthritis (KOA) relies on pain relief medication, such as acetaminophen, nonsteroidal anti-inflammatory drugs (NSAIDs), and intraarticular injection. However gastrointestinal upset and dose dependency are frequent problems with these medications. For non-pharmacological intervention, losing weight is strongly recommended, but long-term acceptance or compliance of weight loss is generally poor. In other words, interests in developing alternative approaches for KOA are needed.Among non-pharmacologic interventions, manual therapy is widely used for musculoskeletal conditions. Manual therapy means that doctors or patients only use their hands to control deterioration of function and pain. In the United States, massage is one of the most popular complementary and alternative therapies (8). Every country's manual therapy style may be different, but as a rule it involves massage, joint mobilization, and manipulation. In recent years, several guidelines recommended manual therapy as an adjunct to core treatments $(7,9,10)$. Three randomized controlled trials (RCTs) have demonstrated that manual therapy could reduce pain, alleviate stiffness, and improve physical function (11-13). The most recent systematic review (SR) suggests that manual therapy is effective compared to no intervention (14), but it is no better than medication or placebo for treating pain in patients with KOA. However, the evidence is insufficient, mostly due to excluding non-English publications and other kinds of manipulations such as tuina (a manual therapy of traditional Chinese medicine), for those studies mostly are published in Chinese. Apart from this, meta-analysis is not performed for heterogeneity using standardized assessment tools and adverse events (AEs) are not included.

For KOA patients, the most common symptoms are pain, stiffness, and physical dysfunction, which also affect quality of life (15). The Western Ontario and
McMaster Universities Osteoarthritis Index (WOMAC) is a self-report questionnaire for $\mathrm{OA}$ of the hip or knee, with higher scores indicating more serious pain, poorer physical function, and increased stiffness. It has been widely used as a tool by clinical investigators to assess patients with KOA. Since it was published in 1988, it has been translated and validated in many languages (16-22). The reliability, validity, and sensitivity to the change in the physical condition of KOA patients has been proven (16). Focusing on those important aspects of clinical outcomes, we performed an updated systematic review and meta-analysis to critically evaluate the effectiveness as well as AEs of manual therapy for KOA.

\section{Methods}

\section{Study Selection}

All eligible studies were randomized controlled trials (RCTs). After duplicates were removed, abstracts of all articles were independently screened based on predefined inclusion criteria. There were no limitations on the participants' age, country, gender, and sample size. The following selection criteria were applied: 1) patients, a clear diagnosis of KOA; 2 ) intervention, including manual therapy, massage, tuina, manipulation, joint mobilization, and osteopathy.

Studies were excluded: 1) if the studies were not RCTs; 2) if the outcome was not assessed by WOMAC; 3 ) if the intervention was multimodal treatment such as manual therapy combined with pharmacologic; and 4) if participants underwent surgery.

\section{Data Sources and Searching Strategy}

Relevant studies were retrieved from the following electronic databases up to October 2015: PubMed, EMBASE, Cochrane Library, China Knowledge Resource Integrated Database (CNKI), Weipu Database for Chinese Technical Periodicals (VIP), CBM, and Wanfang Data, using all the possible Medical Subject Headings (MeSH) and keywords: (manual ther* OR musculoskeletal manipulation OR manipulat* OR joint mobili* OR tuina OR massage OR osteopath*) AND (osteoarthritis, knee OR gonarthrosis OR osteoarthrosis OR osteoarthrit* OR osteoarthropathy OR arthralgia). The search strategy consisted of 3 groups of terms. The first terms were osteoarthritis, gonarthrosis, osteoarthrosis, osteoarthropathy, or arthralgia. The second terms were musculoskeletal manipulation, manual therapy, joint mobilization, tuina, massage, or osteopathy, and the last term was random. We combined these terms for 
the search. There was no limitation to study publication status or language. The three main database search strategies were presented in Appendix I.

\section{Study Selection and Date Abstraction}

We scanned all records by title and abstract to identify relevant studies. Then full text articles were retrieved for further analysis. Two researchers (YLC and $\mathrm{HYH}$ ) independently conducted study selection and extracted data of studies meeting the predefined criteria. The data of every study included the first author, publication year, sample size, participants' mean age, outcome assessment, duration of treatment, and type of study. If there were disagreements, the authors reached consensus by discussing them with the third author (YXZ).

\section{Methodological Quality Assessment}

Two researchers (XZW and TL) independently assessed the methodological quality of each article using the PEDro scale. The PEDro scale is based on the Delphi list and has been reported to have sufficient reliability for RCTs of physical therapy in systematic reviews (23). The PEDro scale consists of 11 items, including (1) specified eligibility criteria of studies, (2) random allocation of studies, (3) concealed allocation, (4) similarity between groups at baseline, (5) blinding of all subjects, (6) blinding of all therapists, (7) blinding of all assessors, (8) less than $15 \%$ dropouts, (9) intention to treat analysis, (10) statistical comparisons between-group, and (11) point measures and variability data. Item 2 through 11 were used to calculate the PEDro score. Each item was scored as either 1 or 0 according to whether the item was met or not, respectively. The score was summed and a higher score represents a better methodological quality. When the PEDro score exceeds the cut-off point 6, this indicates high quality (23). Disagreement was resolved by discussion with the third review author (JP). If a study ranked as low quality it was still included, but this might reduce our confidence of recommendation.

\section{Data Synthesis and Analysis}

All data were combined and analyzed using Cochrane Collaboration software (Review Manager Version 5.2 for Windows; Copenhagen: The Nordic Cochrane Centre). The WOMAC index including 3 items was used to evaluate hip or knee OA patients, i.e. pain, stiffness, and physical function. Each item consisted of 24 parameters. Higher WOMAC scores indicated greater pain, stiffness, or physical disability. Because of differ- ent subscales of WOMAC measuring the outcomes, and property of continuous variables, we choose the standard mean difference (SMD) to calculate the mean difference (24). The mean changes in outcome measures between the end of the final intervention and the baseline was used to assess the difference between the manual therapy group and control group in the meta-analyses. SMD and $95 \%$ confidence intervals (Cls) were calculated. All the treatment effectiveness estimates summary calculations used a random effects model. For the heterogeneity, we used the $P$ statistic, a quantitative measure to assess it (25). Results with an $P$ of $25 \%$ to $50 \%$ indicated low heterogeneous, $P$ of $50 \%$ to $75 \%$ indicated moderate heterogeneous, and $P$ above $75 \%$ indicated high heterogeneous (25). If the heterogeneous rate is too high, the result of the analysis will be biased. So, when $P>50 \%$, the heterogeneity was moderate or high, we should try to seek out the potential sources of it by sensitivity analysis. In view of different treatment duration, subgroups analyses were performed. AEs were also considered.

\section{Results}

\section{Result of Search}

Seven electronic databases (3 English and 4 Chinese) were searched. Of the 2,249 originally identified publications, 1,078 were excluded for duplication. In the following title and abstract screening, 1,072 studies were excluded for not meeting the included criteria. A further 99 potentially related records were retrieved and reviewed for full text. Of which, 85 studies were excluded because they were not RCTs $(n=3)$, the outcome assessment was not WOMAC ( $n=48)$, they were only abstracts $(n=4)$, they included multimodal treatment $(n=22)$, they were study protocols $(n=4)$, or they had unrelated outcomes $(n=4)$. A final library of 14 articles remained for meta-analysis. The articles selection process is summarized in Fig. 1 .

\section{Study Characteristics}

Of the 14 RCTs, 9 were published in English (13,2629) and 5 were published in Chinese (30-38). All studies were parallel designed RCTs and conducted in a single center. The sample size of the studies ranged from 36 to 120 (total 841, including 233 men and 608 women). All participants were older adults.

Maitlan joint mobilization, Swedish massage, Chinese tuina, self-massage, acupressure, manipulation, and manual stretching were the main KOA techniques 


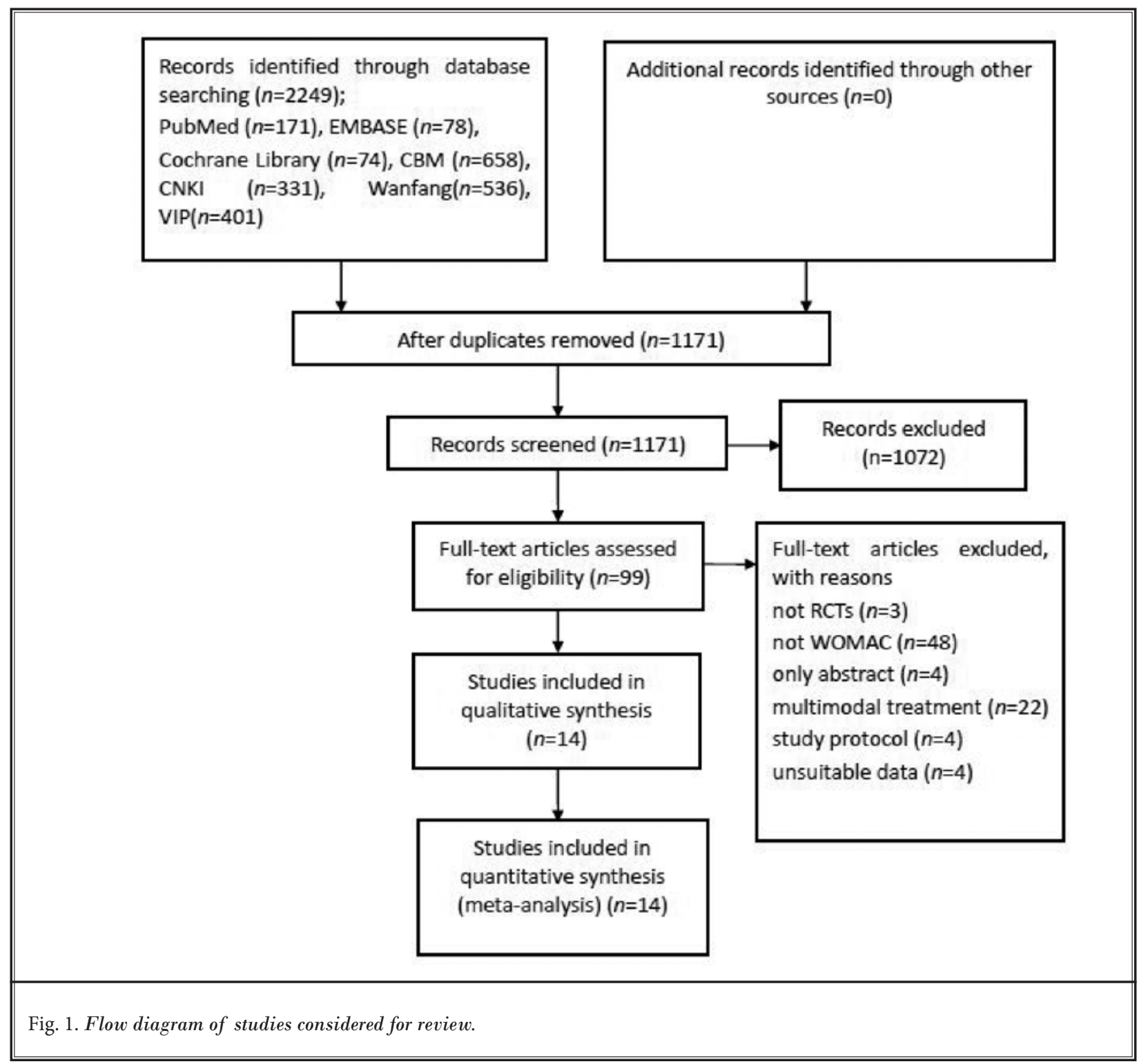

applied. Each time of treatment ranged from about 20 to 60 minutes. Treatment frequency was once a week (28), twice weekly $(27,35,37), 3$ times a week $(26,30,34)$, even 7 times weekly, or ranged from twice a week to once weekly depending on the protocol (13), and treatment duration ranged from 2 to 12 weeks. All study outcomes used WOMAC, while 10 studies (13,26-29,31-33,35,36) described pain, stiffness, function, and total score, one study (37) described pain, stiffness, and function score, and the other 3 studies $(30,34,38)$ described total score. Details of the included studies were listed in Table 1.

\section{Methodological Quality}

The quality score of the included studies was summarized in Table 2. The total score of PEDro scale ranged from 6 to 9 points, with a theoretical maximum of 10 points. Most studies exceeded the cut-off 6 , but they could not be considered high quality with 6 or 7 points for most of them. Only 2 studies scored 9 points. There were serious flaws in terms of concealed allocation, subject blinding, therapist blinding, and assessor blinding. In the remaining items of the PEDro scale, the studies had higher methodological quality. 
Manual Therapy for Knee Osteoarthritis

\begin{tabular}{|c|c|c|c|c|c|c|c|c|}
\hline 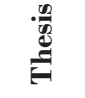 & ஜ & $\stackrel{8}{2}$ & ż & $\stackrel{\circ}{z}$ & $\stackrel{8}{z}$ & ż & 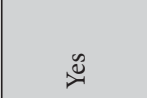 & ż \\
\hline 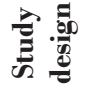 & $\underset{\approx}{5}$ & $\breve{y}_{\breve{q}}$ & $\breve{q}_{\mathscr{q}}$ & $y_{\check{q}}$ & $\breve{y}_{\mathscr{q}}$ & $\underset{\mathscr{Z}}{\breve{y}}$ & 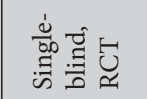 & $\breve{q}$ \\
\hline 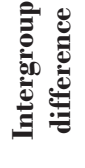 & 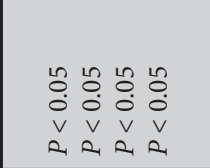 & 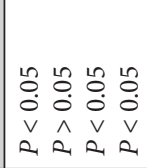 & 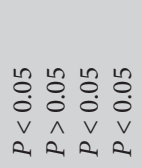 & 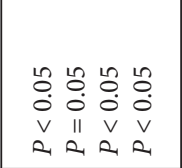 & 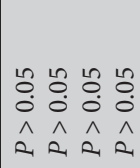 & $\begin{array}{l}\stackrel{0}{0} \\
0 \\
\hat{2} \\
\hat{R}\end{array}$ & 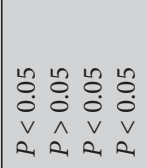 & 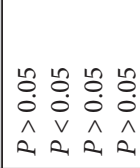 \\
\hline 题 & 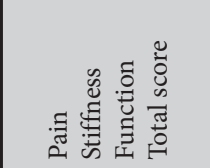 & 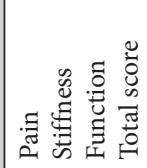 & 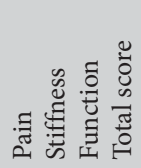 & 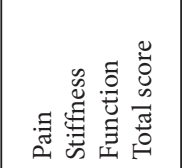 & 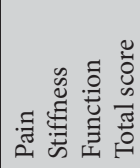 & 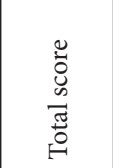 & 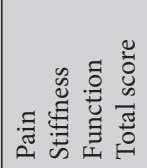 & 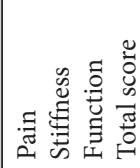 \\
\hline 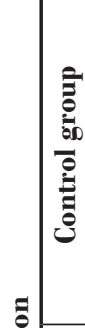 & 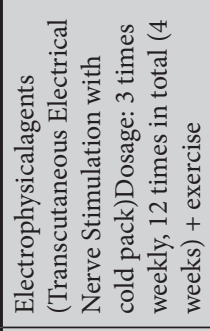 & 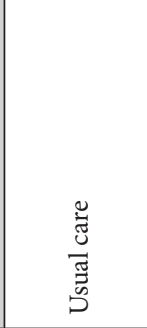 & 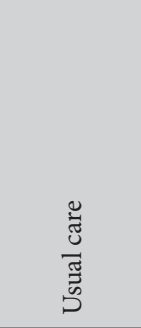 & 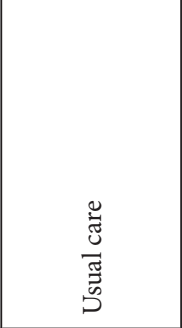 & 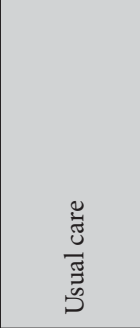 & 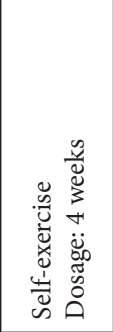 & 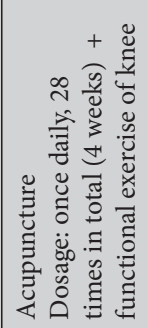 & 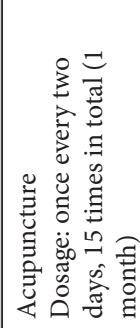 \\
\hline | & 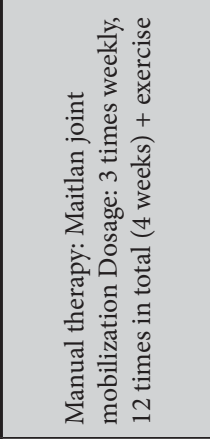 & 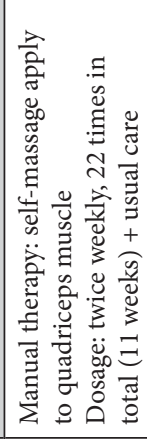 & 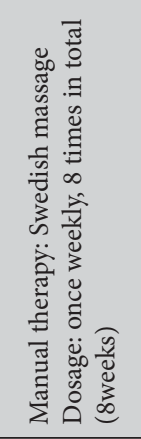 & 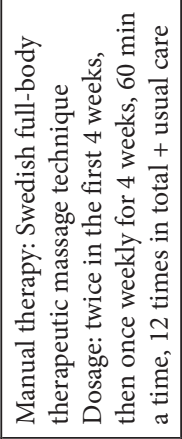 & 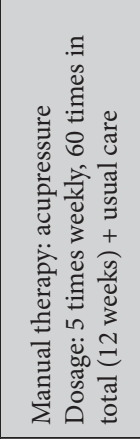 & 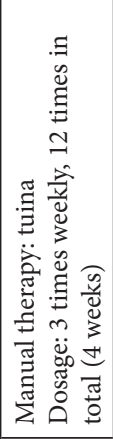 & 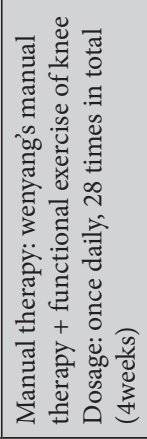 & 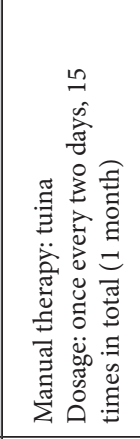 \\
\hline 胥产 & 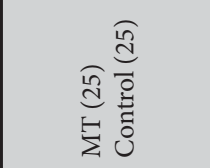 & 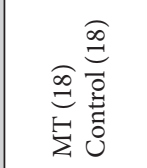 & 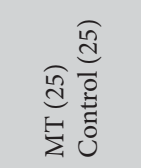 & 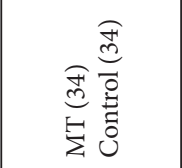 & 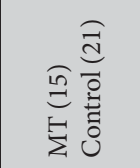 & 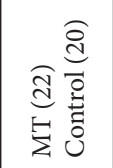 & 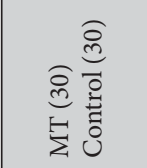 & 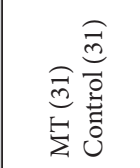 \\
\hline 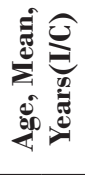 & $\begin{array}{l}8 \\
\dot{1} \\
\stackrel{+}{+}\end{array}$ & $\begin{array}{l}0 \\
\dot{10} \\
0 \\
0 \\
\dot{0} \\
\dot{b}\end{array}$ & 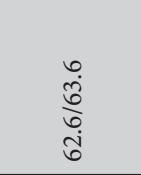 & \begin{tabular}{l}
\multirow{2}{0}{} \\
$\stackrel{0}{0}$ \\
$\stackrel{2}{0}$ \\
$\stackrel{R}{R}$
\end{tabular} & 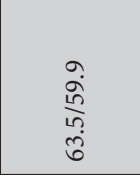 & 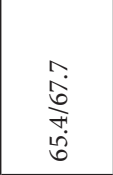 & 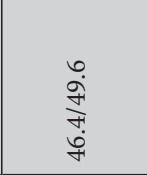 & 号 \\
\hline 题 & $\begin{array}{l}\text { ô } \\
\stackrel{1}{7} \\
\text { d } \\
\text { in }\end{array}$ & $\underset{\substack{\infty \\
\stackrel{\infty}{\rightleftharpoons}}}{\stackrel{\infty}{=}}$ & 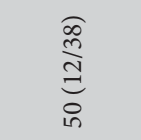 & $\begin{array}{l}\widehat{N} \\
\stackrel{n}{n} \\
\stackrel{0}{0} \\
0 \\
0\end{array}$ & 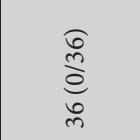 & 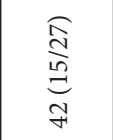 & $\begin{array}{l}\frac{7}{d} \\
\stackrel{8}{6} \\
0\end{array}$ & 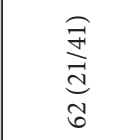 \\
\hline 泀 & 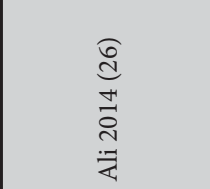 & 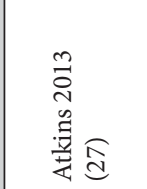 & 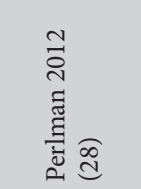 & 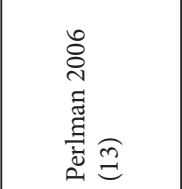 & 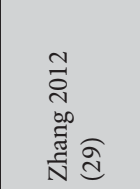 & 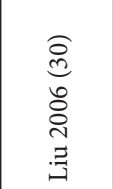 & 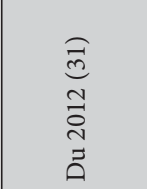 & 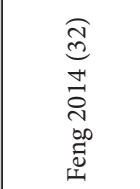 \\
\hline
\end{tabular}


Pain Physician: May/June 2017: 20:229-243

\begin{tabular}{|c|c|c|c|c|c|c|}
\hline$\stackrel{g}{E}$ & 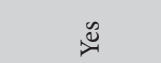 & $\approx$ & 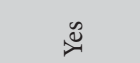 & $\cong$ & $\stackrel{2}{z}$ & $\stackrel{\circ}{z}$ \\
\hline 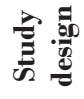 & $\underset{\widetilde{Y}}{\vec{y}}$ & $\underset{\widetilde{x}}{\tilde{y}}$ & $\underset{\widetilde{x}}{5}$ & $\underset{\widetilde{x}}{5}$ & 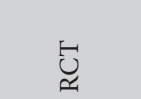 & $\underset{\varkappa}{\breve{y}}$ \\
\hline 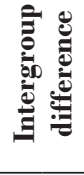 & 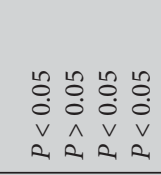 & $\begin{array}{l}\stackrel{0}{0} \\
\dot{0} \\
\hat{2} \\
\text { R }\end{array}$ & 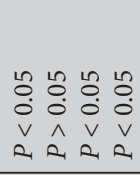 & 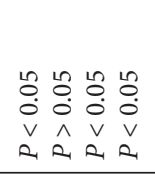 & 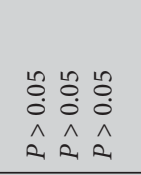 & 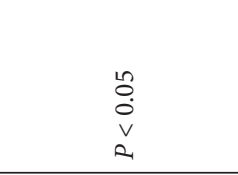 \\
\hline $\begin{array}{l}0 \\
0\end{array}$ & 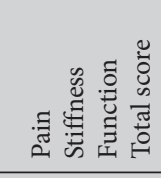 & 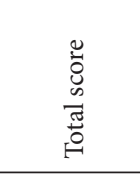 & 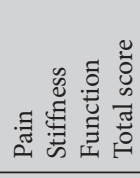 & 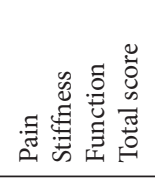 & 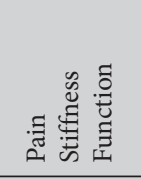 & 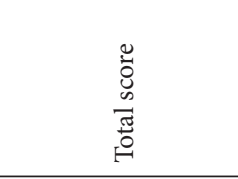 \\
\hline 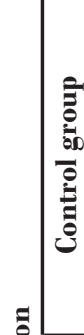 & 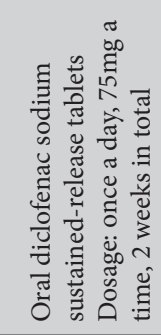 & 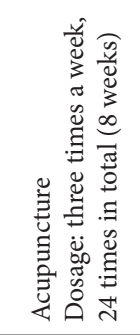 & 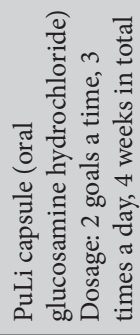 & 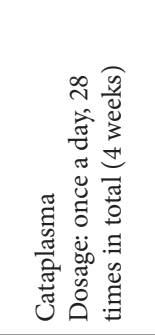 & 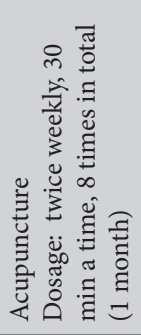 & 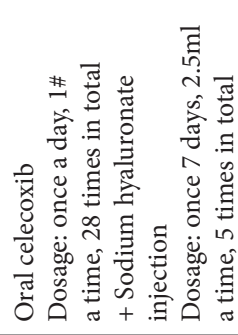 \\
\hline 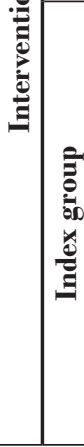 & 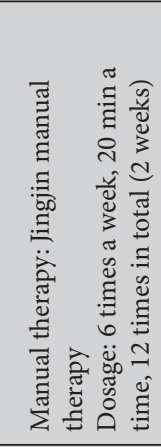 & 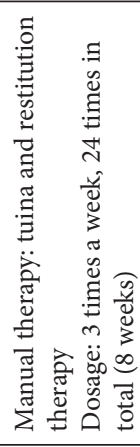 & 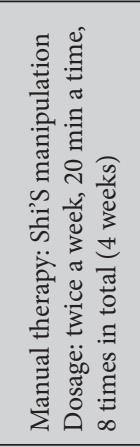 & 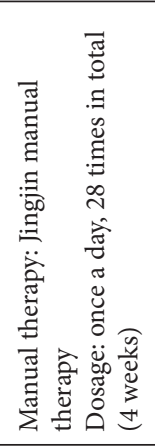 & 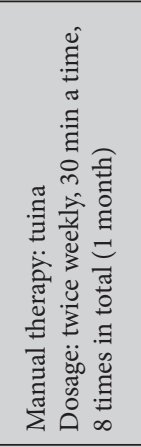 & 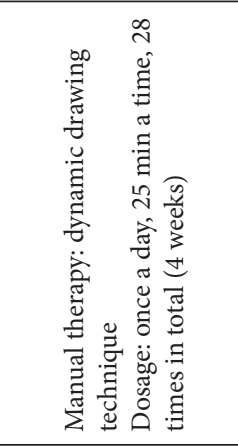 \\
\hline 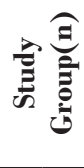 & 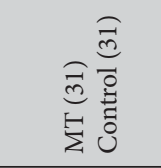 & 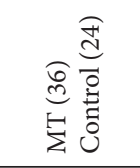 & 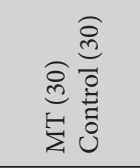 & 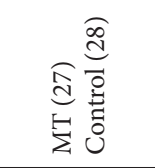 & 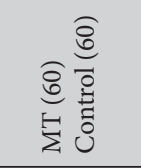 & 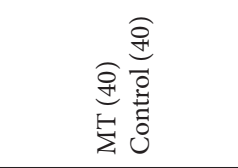 \\
\hline 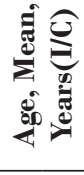 & 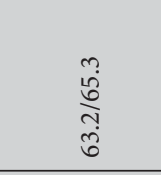 & 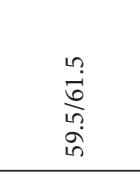 & 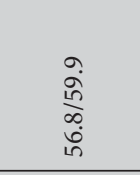 & $\begin{array}{l}\stackrel{0}{\mathrm{j}} \\
\frac{\mathrm{j}}{\mathrm{m}} \\
\stackrel{0}{0}\end{array}$ & $\begin{array}{l}\stackrel{H}{i} \\
\text { o } \\
\infty \\
\vec{b}\end{array}$ & $\begin{array}{l}\vec{b} \\
\stackrel{0}{b} \\
\substack{n \\
i n}\end{array}$ \\
\hline 量 & 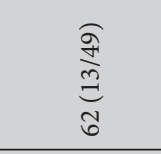 & $\begin{array}{l}\underset{\infty}{\stackrel{\infty}{+}} \\
\underset{d}{0} \\
8\end{array}$ & $\begin{array}{l}\stackrel{\sigma}{\stackrel{7}{\Xi}} \\
\Xi \\
8\end{array}$ & 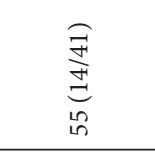 & 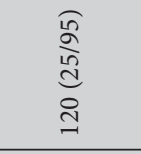 & 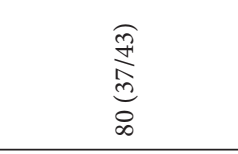 \\
\hline 苞 & 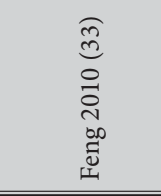 & 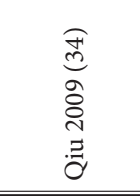 & 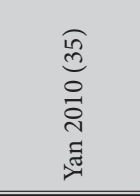 & 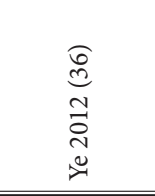 & 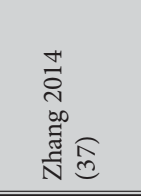 & 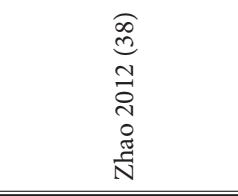 \\
\hline
\end{tabular}




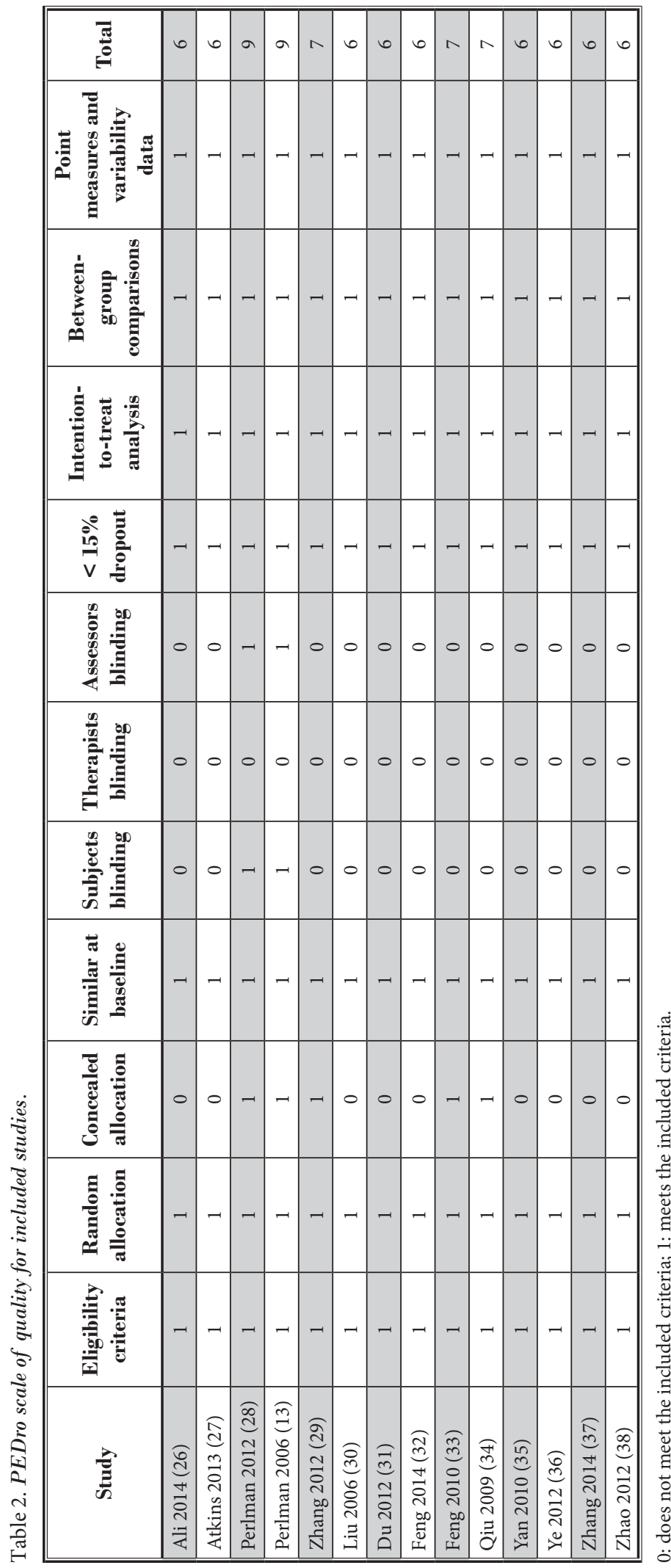

\section{Effects of Interventions}

\section{Effectiveness of Manual Therapy on Pain}

There were 11 studies reporting pain for KOA (13,26-29,31-33,35-37). The meta-analysis showed favorable effects of manual therapy on pain relief $(\mathrm{SMD}=-0.61,95 \% \mathrm{Cl}-0.95--0.28$, $P=0.0003, \mathrm{P}$ for heterogeneity $<0.00001, P=$ $76 \%$ ) (Fig. 2). According to different treatment durations, we conducted subgroup analyses: $<4$ weeks, 4 weeks, and $>4$ weeks. For duration < 4 weeks (SMD $=-0.49,95 \% \mathrm{Cl}-0.85--0.13, P=$ $0.007, P$ for heterogeneity $=0.85, P=0 \%$ ); duration $=4$ weeks $(\mathrm{SMD}=-0.39,95 \% \mathrm{Cl}-0.76--0.02$, $P=0.04, P$ for heterogeneity $=0.005, P=70 \%$ ); duration $>4$ weeks $(\mathrm{SMD}=-0.87,95 \% \mathrm{Cl}-1.55-$ $-0.18, P=0.01, P$ for heterogeneity $=0.003, P=$ $79 \%)$, manual therapy significantly relieve pain (Fig. 3).

\section{Effectiveness of Manual Therapy on Stiffness}

Eleven studies assessed the effectiveness of manual therapy on stiffness for KOA versus control (13,26-29,31-33,35-37). The meta-analysis showed superior effects of manual therapy on stiffness relief $(\mathrm{SMD}=-0.58,95 \% \mathrm{Cl}-0.95--0.21$, $P=0.002, P$ for heterogeneity $<0.00001, P=$ $81 \%$ ) (Fig. 2). In the subgroup analyses, manual therapy did not relieve stiffness for duration < 4 weeks (SMD $=-0.09,95 \% \mathrm{Cl}-0.45--0.26, P=$ $0.60, P$ for heterogeneity $=0.37, P=0 \%$ ), but for duration $=4$ weeks $(\mathrm{SMD}=-0.32,95 \% \mathrm{Cl}-0.59$ $-0.06, P=0.02, P$ for heterogeneity $=0.13, P=$ $42 \%$ ) and duration $>4$ weeks (SMD $=-1.11,95 \%$ $\mathrm{Cl}-1.88--0.34, P=0.005, P$ for heterogeneity $<$ $0.00001, P=87 \%$ ) (Fig. 4).

\section{Effectiveness of Manual Therapy on Physical Function}

Eleven RCTs tested the effectiveness of manual therapy on physical function for KOA versus control (13,26-29,31-33,35-37). The aggregated results of these studies suggest that manual therapy significantly improves physical function $(\mathrm{SMD}=-0.49,95 \% \mathrm{Cl}-0.76--0.22, P=0.0004, P$ for heterogeneity $=0.002, P=65 \%$ ) (Fig. 2). In the subgroup analyses, manual therapy significantly improved physical function for duration $>4$ weeks $(\mathrm{SMD}=-0.72,95 \% \mathrm{Cl}-1.23--0.22$, 
Pain Physician: May/June 2017: 20:229-243

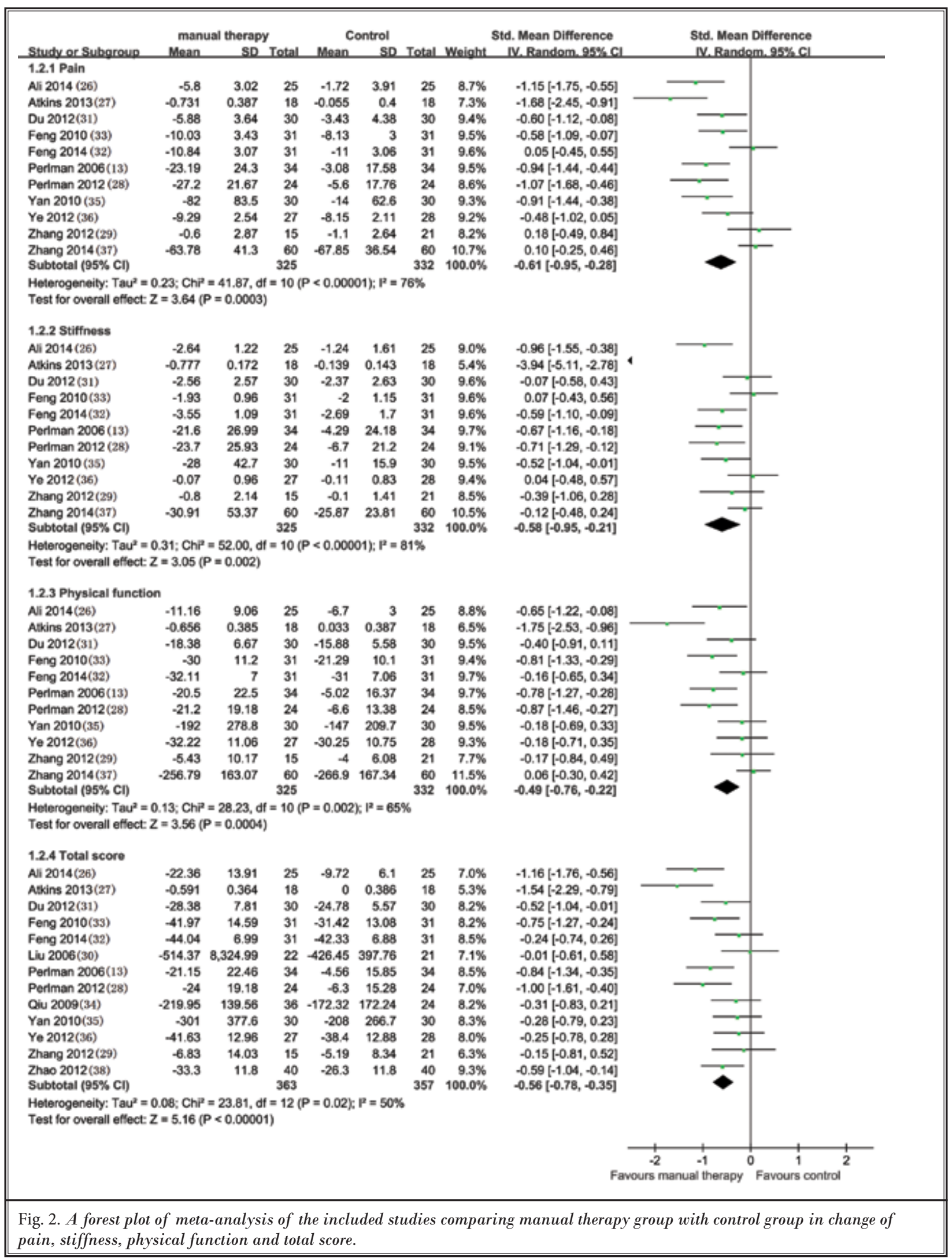


Manual Therapy for Knee Osteoarthritis

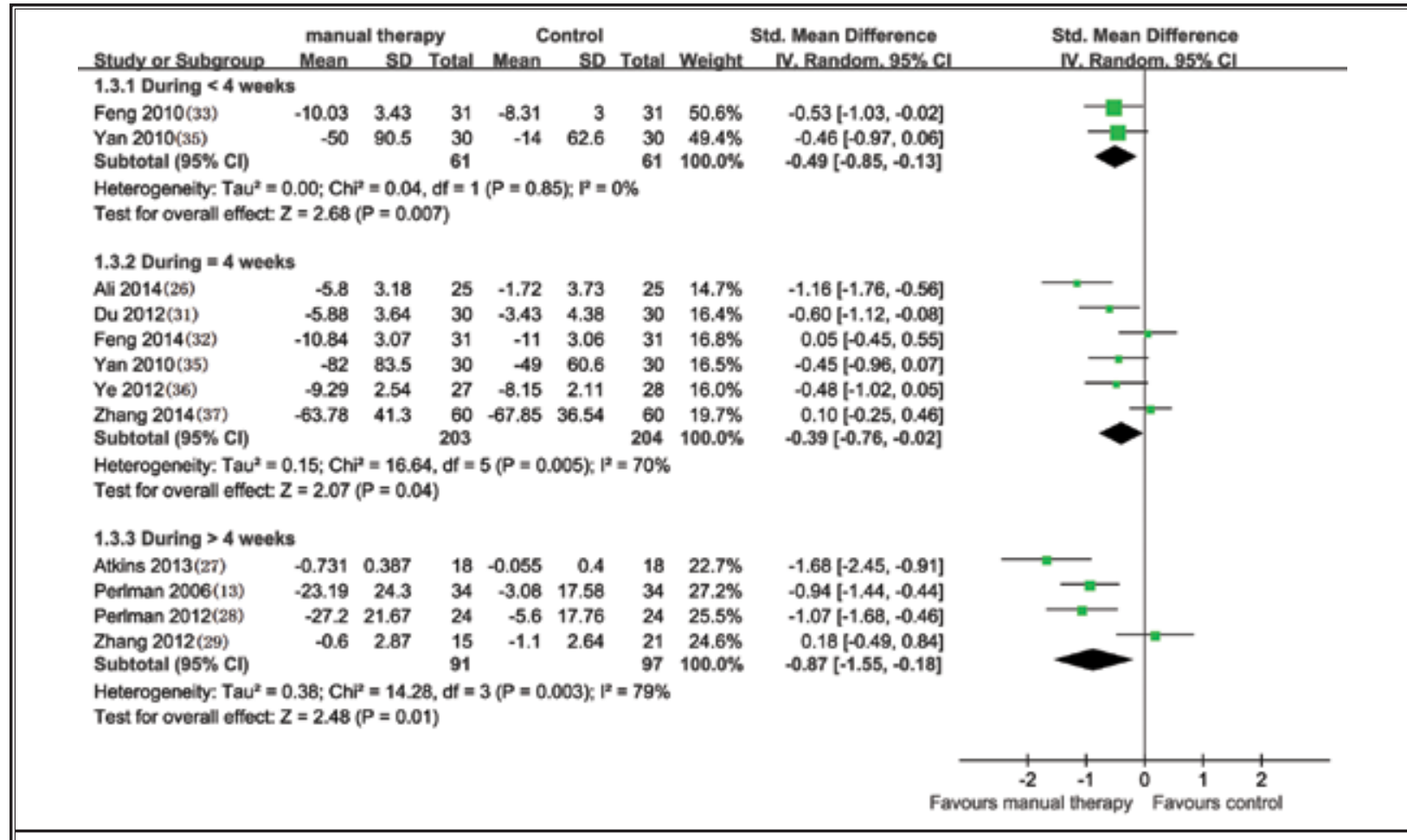

Fig. 3. A forest plot of the subgroup analyses of the included studies comparing manual therapy group with control group in change of pain.

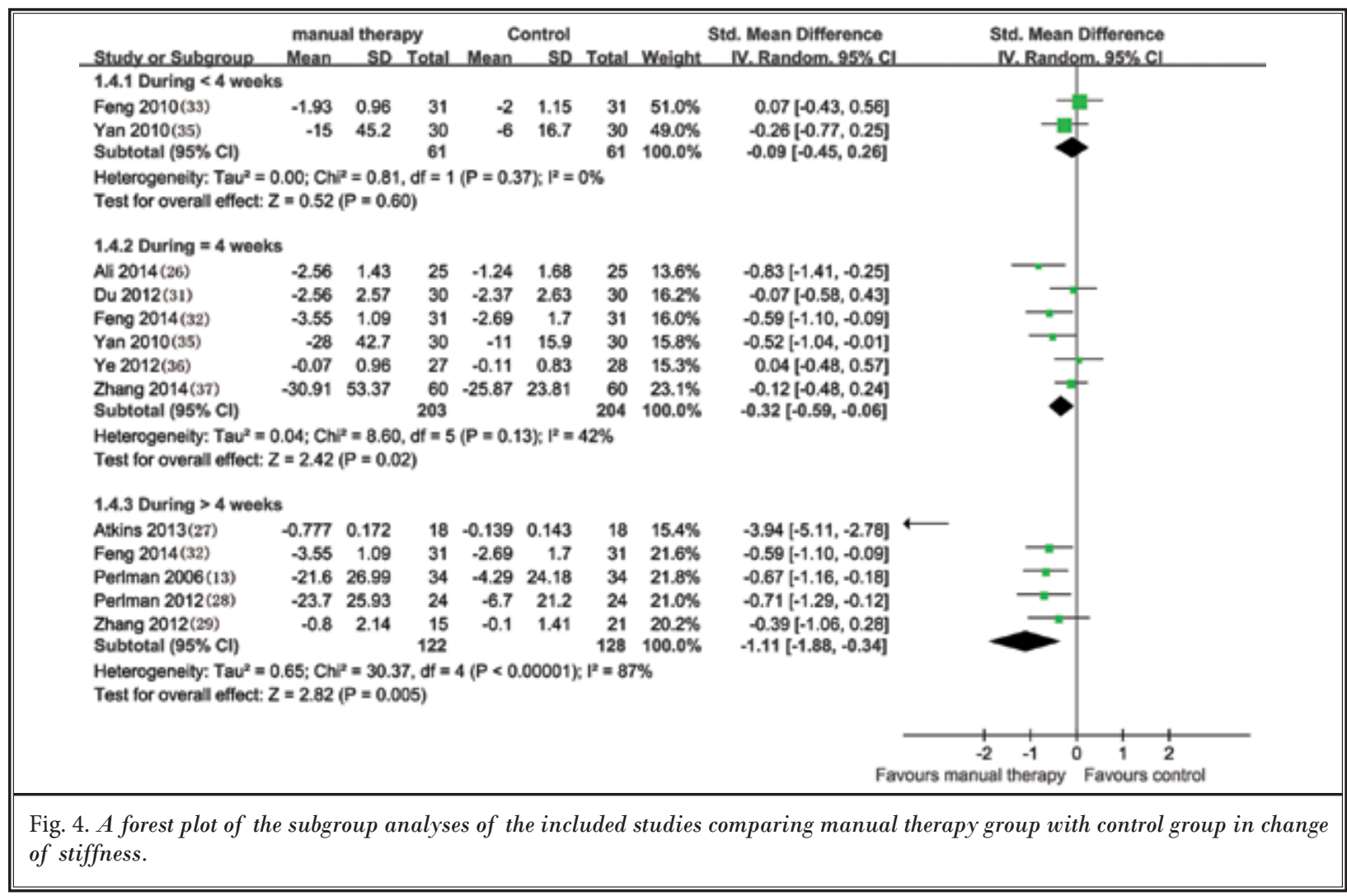


$P=0.005, P$ for heterogeneity $=0.006, P=72 \%)$. But there was no significant improvement for duration < 4 weeks (SMD $=-0.50,95 \% \mathrm{Cl}-1.09--0.08, P=0.09, P$ for heterogeneity $=0.11, P=62 \%)$, nor for duration $=4$ weeks (SMD $=-0.18,95 \% \mathrm{Cl}-0.38-0.01, P=0.07, P$ for heterogeneity $=0.51$ ) (Fig. 5).

\section{Effectiveness of Manual Therapy on Total Score}

Thirteen studies assessed the effect of manual therapy on total score for KOA versus control $(13,26$ $36,38)$. The results showed manual therapy significantly improved KOA (SMD $=-0.56,95 \% \mathrm{Cl}-0.78--0.35, P<$ $0.00001, P$ for heterogeneity $=0.02, P=50 \%$ ) (Fig. 2). In the subgroup analyses, manual therapy was beneficial for the management of KOA for duration < 4 weeks $(\mathrm{SMD}=-0.52,95 \% \mathrm{Cl}-0.97--0.08, P=0.02, P$ for heterogeneity $=0.22, P=33 \%)$, duration $=4$ weeks (SMD $=$ $-0.47,95 \% \mathrm{Cl}-0.72--0.23, P=0.0002, P$ for heterogeneity $=0.09, P=43 \%)$, and $>4$ weeks (SMD $=-0.65,95 \%$ $\mathrm{Cl}-1.04--0.26, P=0.001, P$ for heterogeneity $=0.02, P$ $=63 \%$ ) (Fig. 6).

Finally, a funnel plot was performed for pain ( $n$
$=11$ RCTs), stiffness ( $n=11$ RCTs), physical function ( $n$ $=11$ RCTs), and total score ( $n=13$ RCTs), respectively. However it was difficult to interpret the result of publication bias because of the limitation of RCTs (Fig. 7).

\section{Adverse Events}

Of 14 studies with AEs of manual therapy, only one study reported that one participant felt increased discomfort and refused to complete the assessment. Seven studies $(53.8 \%)$ did not report whether they had AEs or not. The remaining 6 studies (46.2\%) stated that no AEs occurred.

\section{Discussion}

In our review, manual therapy was defined as contact with the soft tissues, bones, and joints with the hands, arms, or elbows of the practitioners to enhance the therapeutic effect. It is one of the oldest forms of treatment, and has been used all over the word since ancient times. It involved mobilization, manipulation, massage, and Chinese tuina techniques.

Recently, there have been an increasing number

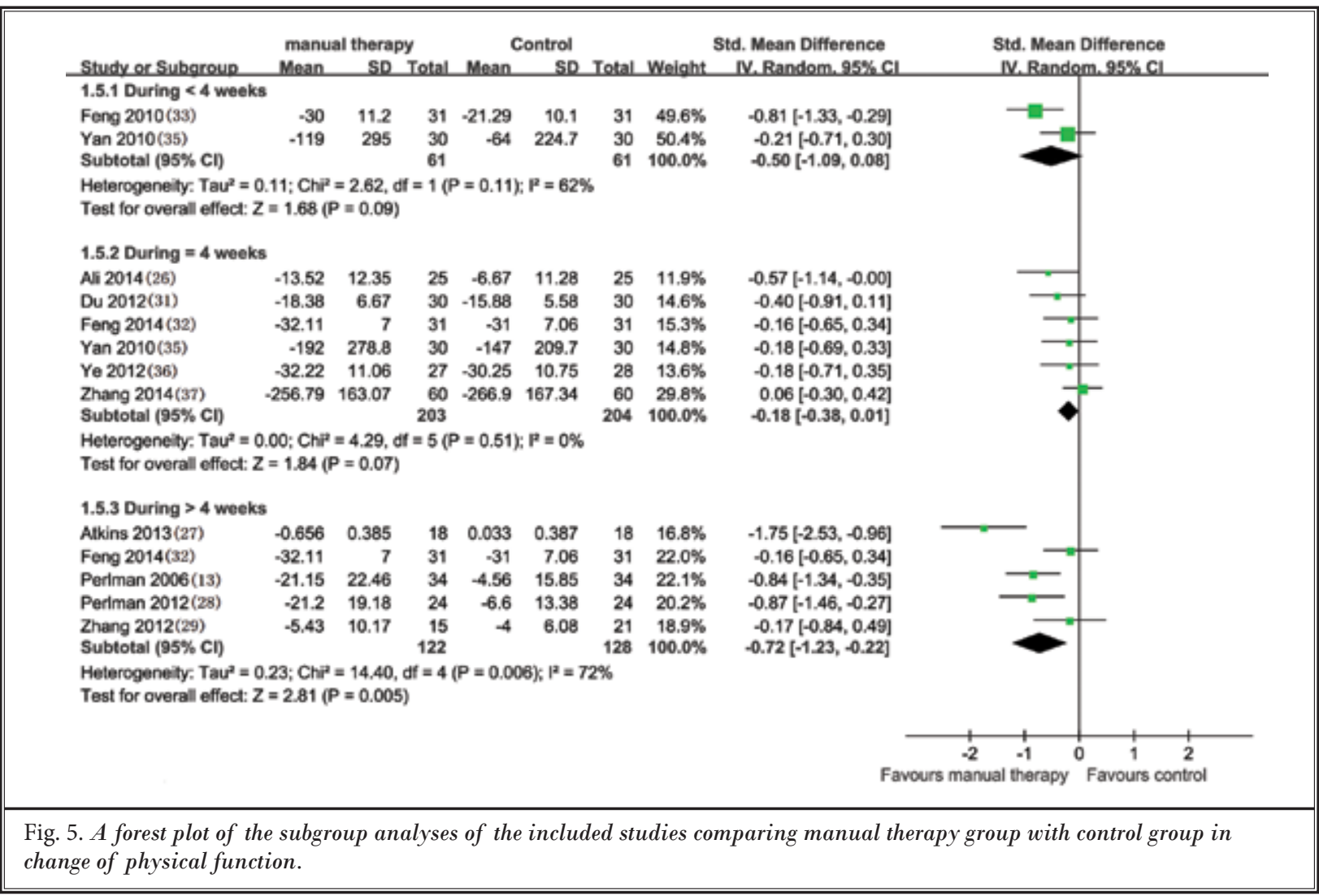


Manual Therapy for Knee Osteoarthritis

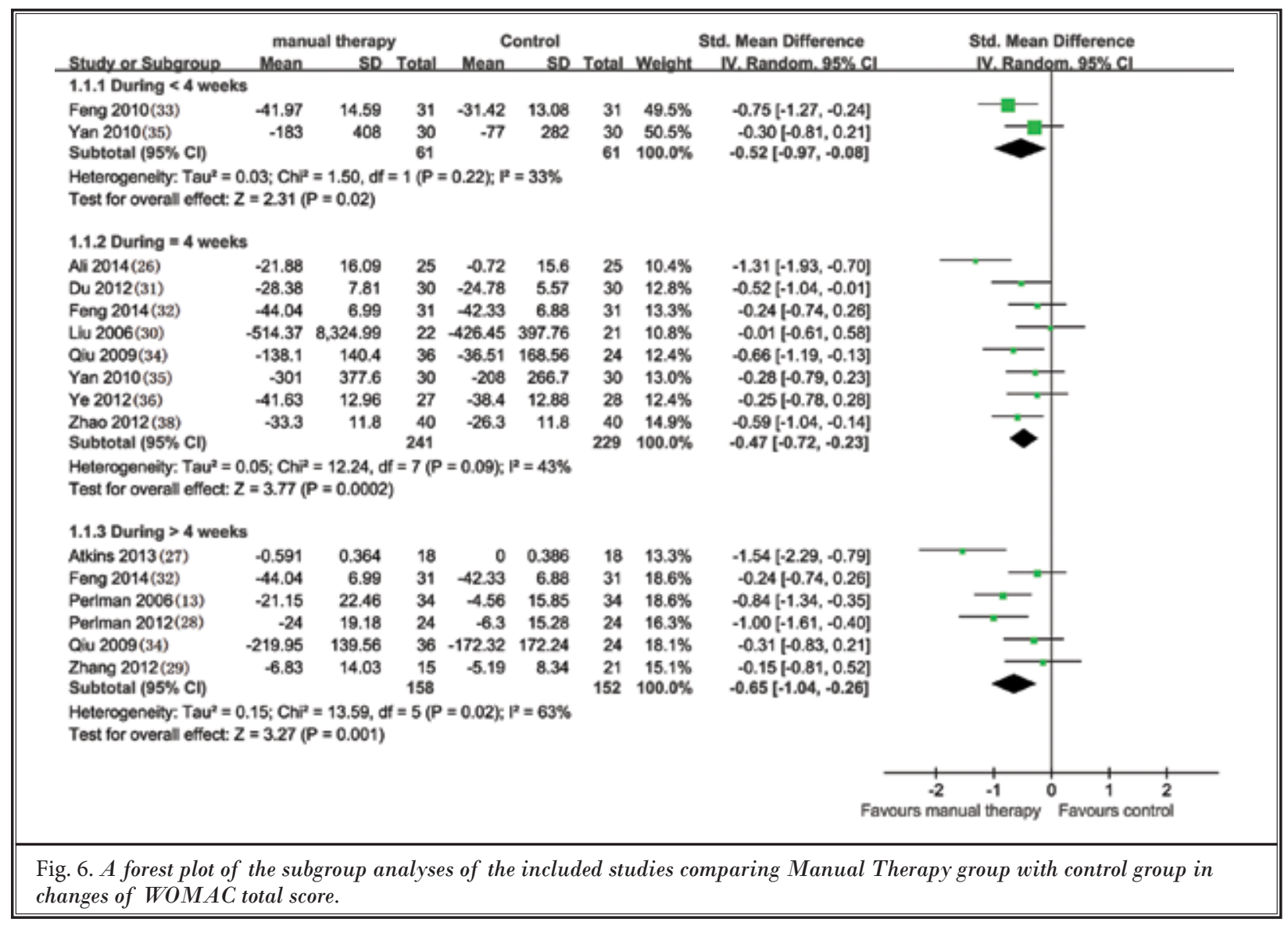

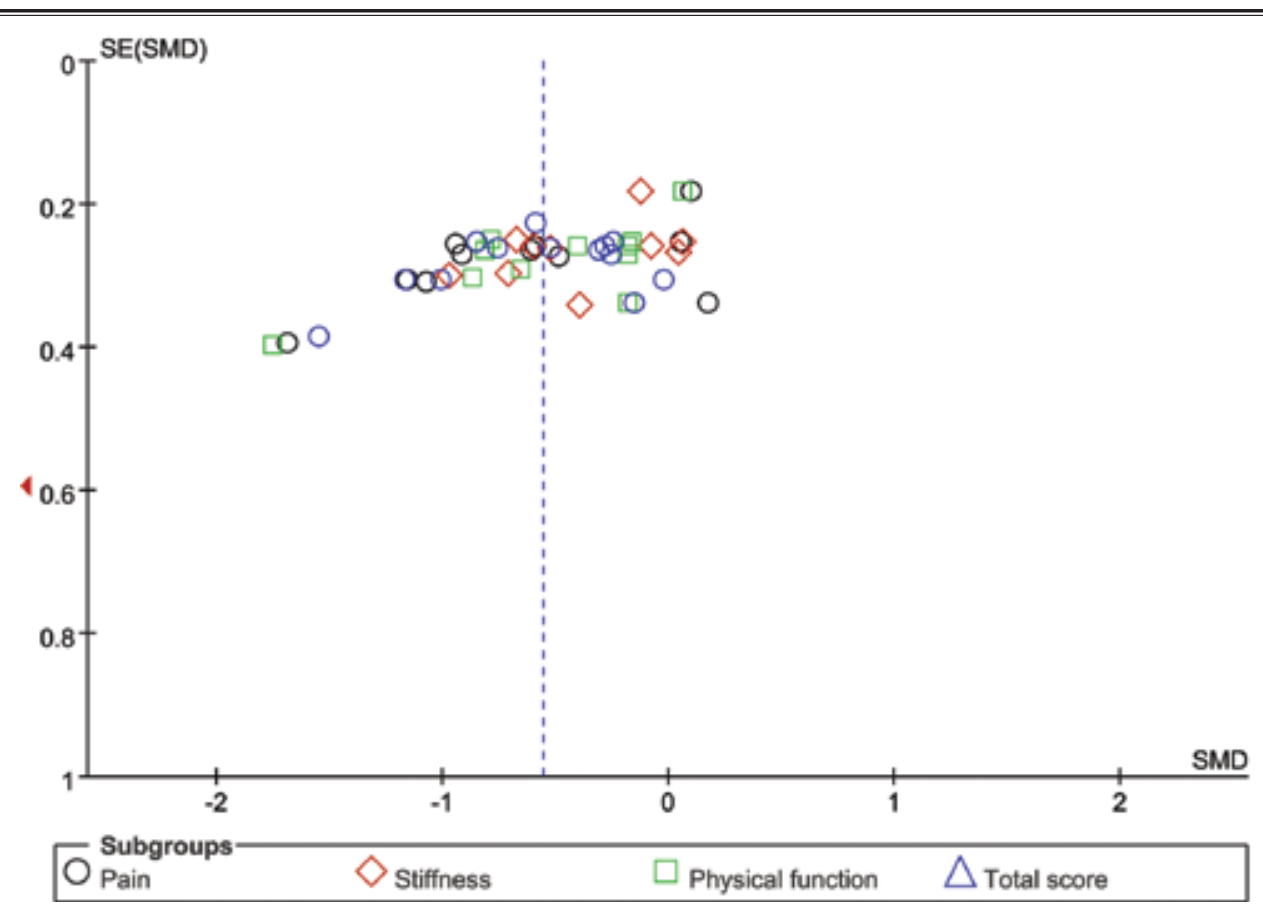

Fig. 7. A Funnel plot for pain, stiffness, physical function, and total score 
of studies aimed at the therapeutic effect of manual therapy for KOA. However, the contribution of manual therapy to manage KOA still remains unclear. There was a systematic review on manual therapy compared to other treatments for KOA in 2011, and the authors did not find any significant evidence regarding the efficacy of manual therapy compared to placebo or meloxicam due to the limited number of studies included and the high risk of bias (14). However, the studies it included were not enough, because they only included studies published in English. We carefully researched relevant clinical outcomes, including pain, stiffness, function, and total score.

The major purpose of this meta-analysis was to update and complete the evidence by adding recent RCTs assessing the effects of manual therapy for patients with KOA. We found that manual therapy significantly reduces pain, alleviates stiffness, and improves physical function in patients with KOA. Our results indicated that manual therapy was beneficial in management of KOA and could be available in rehabilitation programs as a complementary and alternative medicine for patients. But the quality of the included studies was generally poor.

In KOA management, the most important goals are to relieve pain and stiffness, improve physical function and quality of life, and stop the development $(39,40)$. Manual therapy includes many techniques, and is regarded as an effective non-pharmaceutical therapy in the management of OA $(41,42)$. In western countries, the most common treatments are joint mobilization, manipulation, and massage. These treatments are commonly used in clinical practice. The surveys showed that $96 \%$ of Irish physical therapists (43) and $64 \%$ of British therapists (44) use manual therapy in the management of patients with hip and knee OA. In China, the symptoms of KOA belong to "Bi-arthralgia" and "flaccidity," a conception of Traditional Chinese Medicine (TCM) (45). Tuina, a form of manual therapy, is widely used for the treatment of KOA in Chinese hospitals. The specific mechanism of the manual therapy is not clear. One early study reviewed physiological benefits to include increasing blood flow.

Our study showed that manual therapy is effective in lessening pain, relieving stiffness, and improving physical function in patients with KOA. Subgroup analyses suggested that a dose duration of $<4$ weeks manual therapy can reduce pain, $=4$ weeks can reduce pain and stiffness, and $>4$ weeks reduce pain and stiffness, and improve physical function. The positive results of our systematic review were consistent with relevant clinical guidelines. The clinical guidelines from the National Institute for Health and Care Excellence (NICE) in 2008 recommended manipulation and stretching as an adjunct to core treatment in the management of KOA (9). The recommendations for the non-surgical management of hip and knee OA from the Royal Australian College of General Practitioners (RACP) also found evidence for massage therapy, although the evidence grade was D (10). This indicated that manual therapy had a proven effect on KOA. The results of 2 studies supported that finding, and demonstrated the potential for pain-relief through manual therapy in KOA $(46,47)$. Our results were not exactly similar to the latest SR (14). It suggested that manual therapy was no better than a placebo or Meloxicam medication, but massage was effective compared to no intervention. The number of RCTs and included patients was small, so this result based on 3 studies with a high risk of bias was inconclusive. The authors did not perform a quantitative analysis due to the use of different assessment measures evaluating outcomes. Therefore, we pooled the outcome measures evaluated by WOMAC for the meta-analysis. The outcomes of interest consisted of pain, stiffness, and physical function. We included more studies, and performed meta-analysis to assess the effect of manual therapy on KOA according to different durations. Our systematic review provided stronger evidence of reduced pain and stiffness and improved physical function.

In non-pharmacological studies, experience of the therapist was an important factor affecting the outcome. In our review, information about the therapists was not disclosed in most studies. Three studies reported the treatment was delivered by several practitioners $(13,27,28)$, but all the therapists were licensed and experienced. One study used the same therapist for all participants (35). Therapist training was provided in 3 studies $(27,29,31)$. The difference in experience and skills among studies may contribute to discordant results.

We found no significant side effects or AEs associated with manual therapy except that one patient felt uncomfortable. Participants had relatively high adherence in most studies, indicating that the use of manual therapy was safe. According to a recent SR about AEs, massage therapy was not completely safe for all conditions (48). For example, certain spinal manipulation has been reported with serious AEs. However, for KOA, AEs were not encountered and therefore we consider the 
treatment to be safe in this context.

Regarding the long-term effect of manual therapy on OA, so far only 3 studies were reported. The first trial showed that the effective rate of manual therapy was higher than the control group (37). In the second study, although the 3-months recurrence rate of the treatment group was less than the control group, the statistical analysis is of no difference (31). In the third study, there were also no significant difference between manual therapy and control group at 24-weeks follow up (28). Therefore, long-term outcomes of manual therapy on OA need to be further explored.

There were a number of limitations in our review. Firstly, the distorting effects of publication and location bias on systematic reviews and meta-analyses may remain as documented by other studies (49-51). Although we were confident that our search strategy located all relevant data and had performed a funnel plot for the outcomes, it was difficult to explain the publication bias. Another possible source of bias was that some negative studies of manual therapy for KOA may be unpublished. Some of the studies were published in China, where journals are prone to report only positive studies
(52), so our review may be affected by potential poor data. Secondly, most eligible studies had poor quality. Because of inadequate reporting of methodology in these studies, inappropriate allocation, concealment, and shortage of blinding may exaggerate the results of the outcome measures $(53,54)$. Thirdly, our analysis was based on 14 RCTs with a relatively small sample size. Compared with larger sample studies, the treatment effect may be overestimated in smaller studies. Finally, our review may be affected by the high heterogeneity of manual therapy, and AEs were not sufficiently reported.

\section{Conclusions}

In summary, 14 RCTs were analyzed in our systematic review, evaluating the efficacy of manual therapy on pain, stiffness, and physical function in patients with KOA. The positive findings suggested that manual therapy was an effective complementary and alternative treatment for KOA pain, stiffness, and physical function. However, high-quality RCTs with long followup are warranted to confirm our findings.

\section{References}

1. Reginster J. The prevalence and burden of arthritis. Rheumatology 2002; 41:3-6.

2. Kurtais $Y$, Oztuna D, Kucukdeveci AA, Kutlay S, Hafiz M, Tennant A. Reliability, construct validity and measurement potential of the ICF comprehensive core set for osteoarthritis. BMC Musculoskelet Disord 2011; 12:255.

3. Hunter DJ. In the clinic. Osteoarthritis. Ann Intern Med 2007; 147:Itc8-1-itc8-16.

4. Ding C, Cicuttini F, Jones G. Tibial subchondral bone size and knee cartilage defects: Relevance to knee osteoarthritis. Osteoarthritis Cartilage 2007; 15:479-486.

5. Arden N, Nevitt MC. Osteoarthritis: Epidemiology. Best Pract Res Clin Rheumatol 2006; 20:3-25.

6. Oliveria SA, Felson DT, Reed JI, Cirillo PA, Walker AM. Incidence of symptomatic hand, hip, and knee osteoarthritis among patients in a health maintenance organization. Arthritis Rheum 1995; 38:1134-1141.

7. Felson DT, Zhang Y. An update on the epidemiology of knee and hip osteoarthritis with a view to prevention. Arthritis Rheum 1998; 41:1343-1355.
8. Barnes PM, Bloom B, Nahin RL. Complementary and alternative medicine use among adults and children: United States, 2007. Natl Health Stat Rep 2008; 10:1-23.

9. NICE (National Institute of Clinical Excellence). The Care and management of osteoarthritis in adults. www.nice.org. uk/guidance/CG59 2014.

10. RACGP (Royal Australian College of General Practitioners). Guideline for the non-surgical management of hip and knee osteoarthritis. www.racgp.org.au/ guidelines/osteoarthritis 2009.

11. Deyle GD, Henderson NE, Matekel RL, Ryder MG, Garber MB, Allison SC. Effectiveness of manual physical therapy and exercise in osteoarthritis of the knee. A randomized, controlled trial. Ann Intern Med 2000; 132:173-181.

12. Yip YB, Tam AC. An experimental study on the effectiveness of massage with aromatic ginger and orange essential oil for moderate-to-severe knee pain among the elderly in Hong Kong. Complement Ther Med 2008; 16:131-138.

13. Perlman Al, Sabina A, Williams AL, Njike VY, Katz DL. Massage therapy for os- teoarthritis of the knee: A randomized controlled trial. Arch Intern Med 2006; 166:2533-2538.

14. French HP, Brennan A, White B, Cusack T. Manual therapy for osteoarthritis of the hip or knee - a systematic review, Man Ther 2011; 16:109-117.

15. Neogi T, Felson D, Niu J, Nevitt M, Lewis CE, Aliabadi P, Sack B, Torner J, Bradley L, Zhang Y. Association between radiographic features of knee osteoarthritis and pain: Results from two cohort studies. BMJ 2009; 339:b2844.

16. Bellamy N, Buchanan WW, Goldsmith $\mathrm{CH}$, Campbell J, Stitt LW. Validation study of WOMAC: A health status instrument for measuring clinically important patient relevant outcomes to antirheumatic drug therapy in patients with osteoarthritis of the hip or knee. J Rheumatol 1988; 15:1833-1840.

17. Cheung RT, Ngai SP, Lam PL, Chiu JK, Fung EY. Chinese translation and validation of the Kujala scale for patients with patellofemoral pain. Disabil Rehabil 2012; 34:510-513.

18. Hashimoto H, Hanyu T, Sledge CB, Lingard EA. Validation of a Japanese pa- 
tient-derived outcome scale for assessing total knee arthroplasty: Comparison with Western Ontario and McMaster Universities osteoarthritis index (WOMAC). J Orthop Sci 2003; 8:288-293.

19. Tuzun EH, Eker L, Aytar A, Daskapan A, Bayramoglu M. Acceptability, reliability, validity and responsiveness of the Turkish version of WOMAC osteoarthritis index. Osteoarthritis Cartilage 2005; 13:28-33.

20. Stucki G, Meier D, Stucki S, Michel BA, Tyndall AG, Dick W, Theiler R. Evaluation of a German version of WOMAC (Western Ontario and McMaster Universities) Arthrosis Index. Z Rheumatol 1996; 55:40-49.

21. Villanueva I, del Mar Guzman M, Javier Toyos F, Ariza-Ariza R, Navarro F. Relative efficiency and validity properties of a visual analogue vs a categorical scaled version of the Western Ontario and McMaster Universities Osteoarthritis (WOMAC) Index: Spanish versions. Osteoarthritis Cartilage 2004; 12:225-231.

22. Ebrahimzadeh $\mathrm{MH}$, Makhmalbaf $\mathrm{H}$, Birjandinejad A, Keshtan FG, Hoseini HA, Mazloumi SM. The Western Ontario and McMaster Universities Osteoarthritis Index (WOMAC) in Persian speaking patients with knee osteoarthritis. Arch Bone Jt Surg 2014; 2:57-62.

23. Maher CG, Sherrington C, Herbert RD, Moseley AM, Elkins M. Reliability of the PEDro scale for rating quality of randomized controlled trials. Phys Ther 2003; 83:713-721.

24. DerSimonian R, Laird N. Meta-analysis in clinical trials. Control Clin Trials 1986; 7:177-188.

25. Higgins JP, Thompson SG, Deeks JJ, Altman DG. Measuring inconsistency in meta-analyses. BMJ 2003; 327:557-560.

26. Ali SS, Ahmed SI, Khan M, Soomro RR. Comparing the effects of manual therapy versus electrophysical agents in the management of knee osteoarthritis. Pak J Pharm Sci 2014; 27:1103-1106.

27. Atkins DV, Eichler DA. The effects of self-massage on osteoarthritis of the knee: A randomized, controlled trial. Int J Ther Massage Bodywork 2013; 6:4-14.

28. Perlman Al, Ali A, Njike VY, Hom D, Davidi A, Gould-Fogerite S, Milak C, Katz DL. Massage therapy for osteoarthritis of the knee: A randomized dose-finding trial. PLOS ONE 2012; 7:e30248.

29. Zhang $Y$, Shen CL, Peck K, Brismee JM, Doctolero S, Lo DF, Lim Y, Lao L. Training self-administered acupressure ex- ercise among postmenopausal women with osteoarthritic knee pain: A feasibility study and lessons learned. Evid Based Complement Alternat Med 2012; 2012:570431.

30. Liu HB, Zhang JF, Zhong LW, Yang H. Manipulation plus pyrola compound traditional Chinese medicine in treatment of senile osteoarthritis of knee. China J Clin Rehabil 2006; 10:30-32.

31. Du Y. Clinical observation of Wenyang's manual in the treatment of knee osteoarthritis. Chengdu Univ Tradit Chin Med 2012; 10-11.

32. Feng JY. Comparing the clinical effects of manual therapy versus acupuncture for patients with knee osteoarthritis. Asia Pac Tradit Med 2014; 10:80-81.

33. Feng XF. The clinical assessment of Jingjin-type manual therapy treat on knee osteoarthritis with Foot Yangming type-jingjin Meridian. Guangzhou Univ Tradit Chin Med 2010; 12-14.

34. Qiu SZ. The clinical research on knee osteoarthritis with Chinese tuina and restitution. Guangzhou Univ Tradit Chin Med 2009; 27-29.

35. Yan XG. Clinical study on treatment of mild-to-moderate knee osteoarthritis by anti-hyperosteogeny capsule and ShiShi manipulation. Guangzhou Univ Tradit Chin Med 2010; 19-21.

36. Ye XX. The clinical assessment of external treatment on knee osteoarthritis with Taiyang musculature symptoms and signs. Guangzhou Univ Tradit Chin Med 2012; 14-15.

37. Zhang DF, LvYH, Qu JR, Wu YY, Liu YM Comparative study of the clinical efficacy of manipulation and acupuncture in the treatment of knee osteoarthritis. Hainan Med J 2014; 25:661-663.

38. Zhao MY, Liu YB, Wang K, Zheng W, Zhang QA. Clinical research on dynamic drawing technique releasing quadriceps remoris in the treatment of knee osteoarthritis. China J Chin Med 2012; 27:1417-1418.

39. Chyu MC, von Bergen V, Brismee JM, Zhang Y, Yeh JK, Shen CL. Complementary and alternative exercises for management of osteoarthritis. Arthritis 2011; 364319 .

40. Hawker GA, Mian S, Bednis K, Stanaitis I. Osteoarthritis year 2010 in review: Non-pharmacologic therapy. Osteoarthritis Cartilage 2011; 19:366-374.

41. Brakke R, Singh J, Sullivan W. Physical therapy in persons with osteoarthritis. $P M$ \& $R$ 2012; 4:S53-S58.
42. Iversen MD, Rehabilitation interventions for pain and disability in osteoarthritis: A review of interventions including exercise, manual techniques, and assistive devices. Orthop Nurs 2012; 31:103-108.

43. Walsh NE, Hurley MV. Evidence based guidelines and current practice for physiotherapy management of knee osteoarthritis. Musculoskelet Care 2009; 7:45-56.

44. Deyle GD, Allison SC, Matekel RL, Ryder MG, Stang JM, Gohdes DD, Hutton JP, Henderson NE, Garber MB. Physical therapy treatment effectiveness for osteoarthritis of the knee: A randomized comparison of supervised clinical exercise and manual therapy procedures versus a home exercise program. Phys Ther 2005; 85:1301-1317.

45. Wang X, Cao Y, Pang J, Du J, Guo C, Liu T, Wei S, Zheng Y, Chen R, Zhan H. Traditional chinese herbal patch for shortterm management of knee osteoarthritis: A randomized, double-blind, placebo-controlled trial. Evid Based Complement Alternat Med 2012; 2012:171706.

46. Messier SP, Loeser RF, Miller GD, Morgan TM, Rejeski WJ, Sevick MA, Ettinger WH, Jr., Pahor M, Williamson JD. Exercise and dietary weight loss in overweight and obese older adults with knee osteoarthritis: The Arthritis, Diet, and Activity Promotion Trial. Arthritis Rheum 2004; 50:1501-1510.

47. Pollard H, Ward G, Hoskins W, Hardy $K$. The effect of a manual therapy knee protocol on osteoarthritic knee pain: A randomised controlled trial. J Can Chiropr Assoc 2008; 52:229-242.

48. Yin P, Gao N, Wu J, Litscher G. Adverse events of massage therapy in pain-related conditions: A systematic review. Evid Based Complement Alternat Med 2014; 2014:480956.

49. Ernst E, Pittler MH. Alternative therapy bias. Nature 1997; 385:480.

50. Egger M, Smith GD. Bias in location and selection of studies. BMJ 1998; 316:61-66.

51. McAlindon TE, Bannuru RR, Sullivan MC, Arden NK, Berenbaum F, Bierma-Zeinstra SM, Hawker GA, Henrotin Y, Hunter DJ, Kawaguchi H, Kwoh K, Lohmander S, Rannou F, Roos EM, Underwood M. OARSI guidelines for the non-surgical management of knee osteoarthritis. Osteoarthritis Cartilage 2014; 22:363-388.

52. Vickers A, Goyal N, Harland R, Rees R. 
Do certain countries produce only positive results? A systematic review of controlled trials. Control Clin Trials 1998; 19:159-166.

53. Schulz KF, Chalmers I, Hayes RJ, Alt- man DG. Empirical evidence of bias. Dimensions of methodological quality associated with estimates of treatment effects in controlled trials. JAMA 1995; 273:408-412.
54. Pildal J, Hrobjartsson A, Jorgensen KJ, Hilden J, Altman DG, Gotzsche PC. Impact of allocation concealment on conclusions drawn from meta-analyses of randomized trials. Int J Epidemiol 2007; 36:847-857. 
